\title{
On the Bottom Magnetic Fields of Millisecond Pulsars
}

\author{
Chengmin Zhang \\ School of Physics, The University of Sydney, NSW 2006, Australia
}

\begin{abstract}
The magnetic field strengths of most millisecond pulsars (MSPs) are about $10^{8-9}$ gauss. The accretion-induced magnetic field evolution scenario here concludes that field decay is related to the accreted mass, that the minimum or bottom field stops at about $10^{8}$ gauss for Eddington-limited accretion, and scales with the accretion rate as $\dot{M}^{1 / 2}$. The possibility of low field ( $\sim 10^{7}$ gauss) MSPs has been proposed for future radio observations.
\end{abstract}

\section{Introduction and Model}

The low magnetic field (MF) and fast spin of MSPs are ascribed to mass accretion in binary systems. The accretion-induced neutron star magnetic field (NSMF) decay models (e.g. Romani 1990; Cheng \& Zhang 1998), are based on the observational evidence that NSMF decays in the binary accretion phase (Shibazaki et al. 1989). Moreover, van den Heuvel \& Bitzaraki (1995) discovered that the increased amount of mass accreted leads to decay of the NSMF, and the "bottom" field strength of about $10^{8}$ gauss is also implied.

Based on the accretion-induced field decay in the slab geometry approximation proposed by Cheng \& Zhang (1998), we extend it to the the spherical geometry structure of the accreted neutron star illustrated in Figure 1 of Cheng \& Zhang (1998). We assume that the magnetic field lines are frozen in the entire NS crust with homogeneous average mass density. Under the condition of incompressible fluid approximation as well as the constant crustal volume assumption, the piled accreted matter will cause the expansion of the volume of the polar zone by $\delta V_{p}=\frac{\dot{M} \delta t}{\rho}-A_{p} \delta H$, where $A_{p}$ is the surface area of the polar zone, $\rho$ is the average density of the crust, $\dot{M}$ is the accretion rate, $\delta t$ is a particular accretion duration, $H$ is the thickness of the crust and $\delta H / H$ is the fraction of the thickness dissolving into the core which is defined by $\frac{\delta H}{H}=\frac{\dot{M} \delta t}{M_{c r}}$, where $M_{c r}=4 \pi R^{2} \rho H$ is the crust mass. The expansion of the polar zone will dilute the magnetic flux density if the conservation of the magnetic flux is preserved, so $\delta\left(B A_{p}\right)=0$, and the area $A_{p}$ of the accretion polar patch can be accurately expressed as (Shapiro \& Teukolsky 1983) $A_{p}=2 \pi R^{2}\left(1-\cos \theta_{c}\right), \sin ^{2} \theta_{c}=\frac{R}{R_{A}}$, where $\theta_{c}$ is the angle between field line of the star surface and polar axis, $R_{A}$ is the Alfvén radius, $R_{A}=3.2 \times 10^{8}(\mathrm{~cm}) \dot{M}_{17}^{-2 / 7} \mu_{30}^{4 / 7}\left(\frac{M}{M_{\odot}}\right)^{-1 / 7}$, where $M$ is the neutron star mass, $\dot{M}_{17}$ is the accretion rate in units of $10^{17} \mathrm{~g} \mathrm{~s}^{-1}$, and $\mu_{30}$ is the magnetic moment in units of $10^{30} \mathrm{G} \mathrm{cm}^{3}$. Therefore, using the specified 
relation $\delta V_{p}=H \delta A_{p}$ and the equation of conservation of magnetic flux, we get $\delta V_{p}=-V_{p} \frac{\delta B}{B}$. Connecting the above equations we obtain the MF evolutionary equation $A_{p} \delta B /\left[\left(2 \pi R^{2}-A_{p}\right) B\right]=\dot{M} \delta t / M_{c r}$. Considering the initial condition $B(t=0)=B_{0}$, we have

$$
B / B_{f}=\left\{1-\left[C \exp \left(-\frac{2 \Delta M}{7 M_{c r}}\right)-1\right]^{2}\right\}^{-7 / 4}, C=1+\sqrt{1-\left(\frac{B_{f}}{B_{0}}\right)^{4 / 7}},
$$

where $\Delta M=\dot{M} t$ and $B_{f}$ is the magnetic field defined by the Alfvén radius matching the star radius, i.e., $R_{A}\left(B_{f}\right)=R$, which is also the minimum or bottom field strength (van den Heuvel \& Bitzaraki 1995),

$$
B_{f}=1.32 \times 10^{8}\left(\frac{\dot{M}}{\dot{M}_{E d d}}\right)^{1 / 2}\left(\frac{M}{M_{\odot}}\right)^{1 / 4} R_{6}^{-5 / 4} \mathrm{G},
$$

where $\dot{M}_{E d d}$ is the Eddington accretion rate and $R_{6}$ is NS radius in units of $10^{6}$ $\mathrm{cm}$. If $\Delta M=10^{-5} \sim 10^{-4} M_{\odot}$, the MF evolutionary equation can be simplified as $B=B_{0} /\left(1+\frac{\Delta M}{m_{B}}\right)$ with $m_{B}=\frac{1}{2}\left(B_{f} / B_{0}\right)^{4 / 7} M_{c r} \simeq 5 \times 10^{-4}\left(M_{\odot}\right)\left(M_{c r} / 0.2 M_{\odot}\right)$. This is just the same form as the empirical formula of accretion-induced field decay proposed by Shibazaki et al. (1989).

\section{Conclusions}

The main conclusions are summarized in the following. NSMF decays in the binary accretion phase, in a manner inversely correlated with the mass accreted from the companion. The bottom field of MSPs is determined by the condition that magnetospheric radius equal the star radius, which is proportionally related to the accretion rate as $\dot{M}^{1 / 2}$ (White \& Zhang 1997). The bottom field of $\mathrm{Z}$ sources (accreting at Eddington rate) is about $\sim 10^{8}$ gauss, and the bottom field of Atoll sources (1\% of Eddington accretion rate) will correspond to $\sim 10^{7}$ gauss. Such low field MSPs have not yet been discovered in radio observations. The final state of the NS is constrained by the system parameters, such as star radius, star mass and mass accretion rate, which has nothing to do with the initial field or period, which is why the almost homogeneous field distributions of MSPs from $\sim 10^{8} \mathrm{G}$ to $\sim 10^{9} \mathrm{G}$ do not follow the field distributions of the normal pulsars from $\sim 10^{11} \mathrm{G}$ to $\sim 10^{15} \mathrm{G}$.

\section{References}

Cheng, K. S., \& Zhang, C. M. 1998, A\&A, 337, 441

Romani, R. W. 1990, Nature, 347, 741

Shapiro, S. L., \& Teukolsky, S. A. 1983, Black Holes, White Dwarfs and Neutron Stars, (New York: Wiley)

Shibazaki, N., \& Murakami, T., \& Shaham, J., \& Nomoto, K. 1989, Nature, 342,656

van den Heuvel, E. P. J., \& Bizaraki, O. 1995, A\&A, 297, L41

White, N., \& Zhang, W. 1997, ApJ, 490, L87 